\title{
Efficacy of the treatment with dapagliflozin and metformin compared to metformin monotherapy for weight loss in patients with class III obesity: a randomized controlled trial
}

\author{
Aldo Ferreira-Hermosillo ${ }^{1}$, Mario Antonio Molina-Ayala², Diana Molina-Guerrero', Ana Pamela Garrido-Mendoza', \\ Claudia Ramírez-Rentería ${ }^{1}$, Victoria Mendoza-Zubieta², Etual Espinosa ${ }^{2}$ and Moisés Mercado ${ }^{1 *}$
}

\begin{abstract}
Background: Mexico has one of the highest prevalence rates of obesity worldwide. New pharmacological strategies that focus on people with class III obesity are required. Metformin and dapagliflozin are two drugs approved for the treatment of diabetes. Beyond its effects on glucose, metformin has been suggested by some studies to result in weight loss. Therapy with dapagliflozin is associated with a mild but sustained weight loss in patients with diabetes.

The primary outcome of the study is to determine if the combined treatment with dapagliflozin and metformin is more effective than monotherapy with metformin for weight loss in patients with class III obesity and prediabetes or diabetes who are awaiting bariatric surgery (including those patients who do have surgery). We also aimed to assess the effect of this combined treatment on waist circumference, triglycerides, blood pressure, and inflammatory cytokines.

Methods: This randomized phase IV clinical trial will include patients with diabetes or prediabetes who are between the ages of 18 and 60 years and exhibit grade III obesity (defined as body mass index $\geq 40 \mathrm{~kg} / \mathrm{m}^{2}$ ). Patients using insulin will be excluded. Subjects will be randomized to one of two groups as follows: 1) metformin tablets $850 \mathrm{mg}$ PO bid or 2) metformin tablets $850 \mathrm{mg}$ PO bid plus dapagliflozin tablets $10 \mathrm{mg} \mathrm{PO} \mathrm{qd}$. The sample size required is 108 patients, which allows for a 20\% dropout rate: 54 patients in the metformin group and 54 in the metformin/dapagliflozin group. All participants will receive personalized nutritional advice during the study. A run-in period of one month will be used to assess tolerance and adherence to treatment regimens. Anthropometric and biochemical variables will be recorded at baseline and at 1, 3, 6, and 12 months. A serum sample to determine glucagon, ghrelin, adiponectin, resistin, interleukin 6, and interleukin 10 will be collected at baseline and before surgery, or at 12 months (whatever happens first).

Adherence to treatment and adverse and secondary events will be recorded throughout the study. An intention-to-treat analysis will be used.

(Continued on next page)
\end{abstract}

\footnotetext{
* Correspondence: mmercadoa@yahoo.com

${ }^{1}$ Unidad de Investigación Médica en Enfermedades Endocrinas, Hospital de

Especialidades Centro Médico Nacional Siglo XXI, Instituto Mexicano del

Seguro Social, Cuauhtémoc 330, Doctores, 06720 Mexico City, Mexico

Full list of author information is available at the end of the article
}

(c) The Author(s). 2020 Open Access This article is distributed under the terms of the Creative Commons Attribution 4.0 International License (http://creativecommons.org/licenses/by/4.0/), which permits unrestricted use, distribution, and reproduction in any medium, provided you give appropriate credit to the original author(s) and the source, provide a link to the Creative Commons license, and indicate if changes were made. The Creative Commons Public Domain Dedication waiver (http://creativecommons.org/publicdomain/zero/1.0/) applies to the data made available in this article, unless otherwise stated. 
(Continued from previous page)

Discussion: Forty-six percent of the patients in our Obesity Clinic have been diagnosed with prediabetes $(32 \%)$ or diabetes (14\%). The use of dapagliflozin in this population could improve weight loss and other cardiovascular factors. This effect could be translated into less time before undergoing bariatric surgery and better control of associated comorbidities.

Trial registration: Clinicaltrials.gov, ID: NCT03968224. Retrospectively registered on May 29, 2019.

Keywords: Metformin, Sodium-glucose transporter 2 inhibitors, Prediabetes, Obesity, Morbid, Type 2 Diabetes Mellitus,

\section{Background}

Dapagliflozin is a selective sodium-glucose cotransporter type 2 inhibitor (iSGLT2) that blocks glucose resorption in the proximal tubule of the kidney, thereby increasing urinary glucose excretion and reducing blood glucose levels. It is currently indicated in the management of patients with type 2 diabetes (T2D) because of its sustained effect on the reduction of glycemia and glycated hemoglobin (HbA1c) [1]. As described by Baker et al. in their systematic review and meta-analysis, dapagliflozin also decreases systolic blood pressure by $4-5 \mathrm{mmHg}$ and results in a $1.7 \mathrm{~kg}$ weight loss (95\% CI 1.33 to 2.11 ) [2]. Furthermore, Jayawardene et al. [3] reported that dapagliflozin increases high-density lipoprotein cholesterol (HDL-c) concentrations by $1.8 \%$ to $4.4 \%$ and decreases triglyceride concentrations by $2.4 \%$ to $6.2 \%$. Dapagliflozin effects in patients with prediabetes and other types of diabetes are currently the subject of intense research [4]. Current meta-analyses report that its adverse effects include an increase in the risk of genital tract infections (OR 3.48, 95\% CI 2.33 to 5.20) [5] and a low risk of euglycemic ketoacidosis [6], among others. Most of these adverse effects are mild and do not require drug withdrawal.

Metformin is a biguanide that acts via both adenosine monophosphate-activated protein kinase (AMPK)dependent and AMPK-independent mechanisms, inhibiting enzymes related to gluconeogenesis and lipogenesis, while also having antineoplastic effects, delaying the aging process, and regulating gut microbiota [7]. Besides being the most common drug for T2D treatment, it has been reported by some studies as having effects on weight loss. Moguel et al. prescribed metformin for a year at doses of $1500 \mathrm{mg} /$ day to nondiabetic women with a body mass index (BMI) of 25 to $32.9 \mathrm{~kg} / \mathrm{m}^{2}$ (group 1) and doses of $2000 \mathrm{mg} /$ day to women with a BMI 33 to $41.7 \mathrm{~kg} / \mathrm{m}^{2}$ (group 2) in addition to a hypocaloric, carbohydrate-modified diet. They observed that the first group decreased $8.06 \pm 0.96 \mathrm{~kg}(p<0.001)$, and the second group decreased $15.1 \pm 3.3 \mathrm{~kg}(p=0.011)$ [8].

In murine models, the glycosuria effect of dapagliflozin has been associated with a caloric deficit between 80 to $340 \mathrm{kcal} /$ day [9]. As monotherapy in patients with T2D, weight loss ranges from 2 to $3 \mathrm{~kg}$; meanwhile, in a 104-week, randomized, double-blinded study of dapagliflozin versus glipizide as add-on therapies to metformin, weight reductions of up to $5.1 \mathrm{~kg}$ (95\% CI -5.7 to $-4.4 \mathrm{~kg}$ ) without regain (at least for 2 years) have been reported [10]. Other studies have shown that dapagliflozin is as effective as metformin for weight loss. In a 12-week, randomized, parallel-group, double-blind, placebo-controlled study, patients assigned to dapagliflozin $10 \mathrm{mg}$ daily presented a $2.7 \%$ reduction in body weight (95\% IC -3.5 to $-1.8 \%$ ) as compared to a weight loss of $1.7 \%$ in those assigned to the metformin group $(95 \% \mathrm{CI}-2.4$ to $-0.9 \%)$ and $1.2 \%$ in patients assigned to placebo ( -2.0 to $-0.4 \%)$ [11]. In a 48-week, randomized, double-blinded, placebo-controlled, parallel-group study, when used in combination with pioglitazone, dapagliflozin resulted in a $2.3 \mathrm{~kg}$ weight loss $(95 \% \mathrm{CI}-3.37$ to $-1.23 \mathrm{~kg})$ [12].

In a systematic review by Orme et al., in patients previously treated with sulfonylurea, dapagliflozin induced a $1.54 \mathrm{~kg}$ weight loss (95\%CI -2.16 to $-0.92 \mathrm{~kg}$ ), compared to patients treated with GLP-1 (loss: $-0.65 \mathrm{~kg}, 95 \% \mathrm{CI}$ -1.37 to $0.07 \mathrm{~kg}$ ) and a DPP4 inhibitor (gain of $0.57 \mathrm{~kg}$, $95 \%$ CI 0.09 to $1.06 \mathrm{~kg}$ ) [13]. Furthermore, the addition of dapagliflozin in patients already treated with metformin and sitagliptin resulted in a $2.1 \mathrm{~kg}$ reduction (95\% CI -3.2 to $-1 \mathrm{~kg}$ ) compared to placebo [14]. Finally, Bolinder et al. found that the addition of $10 \mathrm{mg}$ dapagliflozin to patients inadequately controlled with metformin resulted in a decrease of $2.08 \mathrm{~kg}$ of total weight $(-2.8$ to $-1.31 \mathrm{~kg}, p<0.001)$ and decreased waist circumference by $1.52 \mathrm{~cm}(2.74$ to $0.31 \mathrm{~cm}, p=0.014)$ after 24 weeks of treatment in a randomized, parallel-group, double-blind, placebo-controlled study [15].

In a meta-analysis, Zhang et al. found that the use of dapagliflozin resulted in $20 \%$ less weight reduction than expected (2.5 kg instead of $3.05 \mathrm{~kg}$ ) [16]. This could be related to the increase in appetite observed in DIO (dietinduced obesity) rats by Devenny et al. [7]. The effect of dapagliflozin in appetite has not been evaluated in humans.

Notably, all studies have been conducted in patients with T2D and a BMI under $40 \mathrm{~kg} / \mathrm{m}^{2}$, so the effects dapagliflozin on weight and other metabolic factors have not been studied in populations with extreme obesity. 
Furthermore, the effects of dapagliflozin on the cytokine profile and on appetite markers like ghrelin have not been studied in this population.

Patients under evaluation for bariatric surgery have been suggested to decrease $10 \%$ of their excess weight to improve the control of some of the comorbidities that are associated with a higher surgical risk such as diabetes, hypertension, or sleep apnea [17]. This also allows evaluating the patients' adherence to medical therapy and their capability to follow a diet. International guidelines suggest the use of drugs for weight loss when BMI is higher than $30 \mathrm{~kg} / \mathrm{m}^{2}$, but only few have been approved due to their adverse effects, including increased blood pressure, anxiety, tachycardia and psychiatric effects [18]. Patients with severe obesity use a daily average of $4.4 \pm 4.1$ medications to control comorbidities such as diabetes, including psychoanaleptics and agents acting on the renin-angiotensin system $(12 \%, 11.3 \%$, and $8.2 \%$ of all medications, respectively) [19]. Therefore, most patients may not be candidates for some of these weight loss drugs, which will limit their chances to undergo surgery. SGLT-2 inhibitors have gained a privileged position in the management of T2D in the last few years due to their ability to control glucose as well as other cardiovascular risk factors including blood pressure, weight, and the lipid profile. Currently, diabetes guidelines place these inhibitors as the first choice for patients with diabetes and high cardiovascular risk [20].

The aim of the study is to determine if combined treatment with dapagliflozin and metformin $(\mathrm{D} / \mathrm{M})$ is more effective than monotherapy with metformin (M) for weight loss in patients with class III obesity and prediabetes or diabetes who are waiting for bariatric surgery.

\section{Methods}

\section{Study hypotheses}

The main hypothesis of the study is that dapagliflozin $10 \mathrm{mg}$ tablets PO qd in combination with metformin $850 \mathrm{mg}$ tablets $\mathrm{PO}$ bid (D/M group) in comparison with metformin alone in doses of $850 \mathrm{mg}$ PO bid (M group) is associated with more weight loss for a maximum of 12 months of treatment (weight will be evaluated at months $1,3,6$, and 12) in patients with prediabetes or T2D and class III obesity who are awaiting bariatric surgery. An exploratory hypothesis is that the combination of $\mathrm{D} / \mathrm{M}$ will also be associated with a decrease in the concentration of pro-inflammatory cytokines such as interleukin-6 (IL-6) and resistin and an increase in antiinflammatory cytokines such as interleukin-10 (IL-10) and adiponectin, as well as increases in serum insulin, glucagon, and ghrelin concentrations in comparison with $\mathrm{M}$ treatment. The secondary hypotheses are that patients in combination therapy with $\mathrm{D} / \mathrm{M}$ will have greater reductions in BMI, systolic blood pressure (SBP) and diastolic blood pressure (DBP), waist circumference (WC), insulin resistance (IR) measured by HOMA-IR, and serum triglycerides (TG) levels in comparison with patients in the M group.

\section{Primary objective}

The primary objective of the study is to assess if combined treatment with $\mathrm{D} / \mathrm{M}$ is more effective than metformin for weight loss in patients with diabetes and prediabetes and class III obesity who are awaiting bariatric surgery at 12 months (including those patients who do have surgery after the required weight loss).

\section{Secondary objective}

An exploratory objective of the study is to assess if the combination therapy with $\mathrm{D} / \mathrm{M}$ is associated with a change in the concentration of pro-inflammatory cytokines such as IL-6 and resistin or in anti-inflammatory cytokines like IL-10 and adiponectin, as well as ghrelin, insulin and glucagon concentrations. Secondary objectives include comparisons among groups of treatment on SBP, DBP, WC, IR, and TG. We also aim to assess the prevalence of adverse effects associated with dapagliflozin use and if participants in both groups decrease the number of medications needed for the control of comorbidities.

\section{Type of study}

This study is an investigator-initiated, controlled, parallel group, randomized controlled trial. The investigators and clinical staff involved in the care of the patients are not blinded. The investigators that register the information into data sheets and those that perform the statistical analysis will be blinded to the treatment provided.

\section{Study population}

Patients with class III obesity (defined as a BMI greater than $40 \mathrm{~kg} / \mathrm{m}^{2}$ ) who present with T2D or prediabetes at the time of screening or are diagnosed with these conditions during screening will be included. Diagnosis will be established according to the most recent American Diabetes Association (ADA) criteria [21]. The participating patients must have been referred for bariatric surgery to The Obesity Clinic at the Hospital de Especialidades Centro Medico Nacional Siglo XXI. The study period will be from August 2018 to August 2020.

\section{Study design}

A convenience sampling will be done for patients previously or currently diagnosed with T2D or prediabetes according to the ADA criteria, who have been referred to the Obesity Clinic of the Hospital de Especialidades Centro Médico Siglo XXI, an academic hospital in Mexico City, Mexico. The schedule of enrollment, 
interventions, and assessments are in accordance with the Standard Protocol Items: Recommendations for Interventional Trials (SPIRIT) Statement as outlined in Fig. 1 [22]. A checklist is also available as supplementary information (Additional file 1).

Patients will be approached and invited to participate in the study when they attend their first clinical appointment (screening visit). At this visit, we will determine an HbA1c in those patients with a previous diagnosis of prediabetes or diabetes to further assess if they qualify for the study. In those patients without previous diagnosis, we will perform an oral glucose tolerance test to determine if they have diabetes or prediabetes.

A full explanation of the purposes of the research will be provided during the screening visit. If the patients agree to participate, the informed consent will be obtained during the baseline visit by one of the investigators. During the baseline visit (a week after screening visit), the investigators will assess patient eligibility against the study inclusion/exclusion criteria. The presence of comorbidities will also be recorded at the baseline visit. The type and dose of drugs used for patient treatment will be recorded at baseline and during follow-up visits.

APGM will generate the allocation sequence for randomization using the computer software Research Randomizer [23]. Additionally, in order to ensure that the groups are comparable, six balanced blocks of participants (18 participants per block, three blocks of participants treated with dapagliflozin, and three blocks of participants treated with metformin and dapagliflozin) will be established. Once randomized, DMA and EEC will enroll participants and will assign them to either group: 1) metformin (M; $1700 \mathrm{mg} /$ day) or 2) metformin (1700 mg/day) and dapagliflozin $10 \mathrm{mg} /$ day (D/M).

Once assigned to the corresponding group, participants will receive an identification code that will be retained throughout the study. The investigators who will perform the data analysis will be blinded to the treatment that the participant is receiving. The investigators will analyze the data at the end of the study. Unblinding is not permissible for investigators involved in the data management. The tolerance to treatments will be assessed a month after the baseline visit. The participants will not be excluded even if they cannot tolerate treatments. If participants want to withdraw from their assigned treatment, they will be invited to continue with nutritional advice for weight loss and with other type of treatment for glucose control (e.g., use of thiazolidinediones, sulfonylureas, or insulin). This information will also be analyzed.

Anthropometric and biochemical variables will be recorded at baseline visit and at follow-up visits: 1, 3, 6, and 12 months. The determination of insulin, glucagon, ghrelin, adiponectin, resistin, IL-6, and IL-10 will be performed at the baseline visit, before surgery, or at 12 months. We aim to explore if those hormones and inflammatory markers change due to weight loss in comparison with those that do not achieve the desired weight loss at 12 months.

Registration of adverse and secondary events will take place from baseline visit and during follow-up visits. For the primary objective of the study, the data will be analyzed at $1,3,6$, or 12 months. The amount of weight loss will be evaluated until 12 months even if participants undergo bariatric surgery or if they cannot decrease weight in the specified time. Data from participants who because of poor glycemic control require treatment with insulin or sulfonylurea will be analyzed during follow-up visits at $1,3,6$, and 12 months as the whole group (intention-to-treat analysis). Participants who did not achieve weight loss during the one-year follow-up will be discharged to their primary care hospital for continuation with dietary treatment as recommended by local guidelines.

\section{Selection criteria}

Inclusion and exclusion criteria are presented in Table 1. Both patients previously diagnosed with T2D or prediabetes and those detected by an oral glucose tolerance test (OGTT) at the screening visit will be included if their $\mathrm{HbA} 1 \mathrm{c}$ is between $5.7 \%$ and $9 \%$. Patients will be required to suspend any other treatment for glycemic control $8 \mathrm{~h}$ before labs and randomization to minimize the impact of such treatment on the inflammatory profile. Those participants with a lack of adherence to medical treatment (defined as consumption of less than $90 \%$ of tablets granted) or less than $80 \%$ of adherence to the nutritional recommendations or who miss more than $20 \%$ of the clinical appointments; those with an adverse effect grade 4 or 5 (assessed with the Common Terminology Criteria for Adverse Effects, CTCAE v 5.0 [24]; those with intolerance to metformin or dapagliflozin evaluated during the run-in period; and those that initiate any other treatment or product for weight loss despite being advised against it will also be considered for analysis. Those participants with genital or urinary tract infection (UTI, detected by symptomatology and evidenced by urinalysis) at the screening visit will be treated with proper antibiotic treatment and invited to participate a week later if the infection is resolved as demonstrated by a new urinalysis.

\section{Nutritional, anthropometrical, and biochemical assessment}

A single nutritionist from the Nutrition Department will evaluate all participants throughout the study. Each participant receives an individualized diet depending on initial weight, ideal weight, and dietary patterns at the time 


\begin{tabular}{|c|c|c|c|c|c|c|}
\hline \multirow[b]{3}{*}{ TIMEPOINT ${ }^{\star \star}$} & \multicolumn{6}{|c|}{ STUDY PERIOD } \\
\hline & \multirow{2}{*}{$\frac{\text { Screening }}{-t_{1}}$} & \multirow{2}{*}{$\frac{\text { Baseline }}{0}$} & \multicolumn{4}{|c|}{ Follow-up (months) } \\
\hline & & & 1 & 3 & 6 & 12 \\
\hline \multicolumn{7}{|l|}{ ENROLLMENT: } \\
\hline Eligibility screen & $x$ & & & & & \\
\hline Informed consent & & $\mathrm{x}$ & & & & \\
\hline Oral Glucose Tolerance Test & $\mathrm{x}$ & & & & & \\
\hline Randomization: Group $M$ or $D / M$ & & $x$ & & & & \\
\hline \multicolumn{7}{|l|}{ INTERVENTIONS: } \\
\hline Dapagliflozin+ Metformin & & $\mathrm{x}$ & $x$ & $\mathrm{x}$ & $x$ & $\mathrm{x}$ \\
\hline Metformin & & $\mathrm{x}$ & $\mathrm{x}$ & $\mathrm{x}$ & $\mathrm{x}$ & $\mathrm{x}$ \\
\hline $\begin{array}{c}\text { Dietary treatment / Nutritionist } \\
\text { evaluation }\end{array}$ & & $\mathrm{x}$ & $\mathrm{x}$ & $\mathrm{x}$ & $\mathrm{x}$ & $\mathrm{x}$ \\
\hline Evaluation of amount of exercise & & $\mathrm{x}$ & $\mathrm{x}$ & $\mathrm{x}$ & $\mathrm{x}$ & $\mathrm{x}$ \\
\hline \multicolumn{7}{|l|}{ ASSESSMENTS: } \\
\hline Medical History/ Demographics & $x$ & & & & & \\
\hline $\begin{array}{l}\text { Recording of type and doses of } \\
\text { treatments }\end{array}$ & & $\mathrm{x}$ & $\mathrm{x}$ & $\mathrm{x}$ & $\mathrm{x}$ & $\mathrm{x}$ \\
\hline Weight & $\mathrm{x}$ & $\mathrm{x}$ & $\mathrm{x}$ & $\mathrm{x}$ & $\mathrm{x}$ & $\mathrm{x}$ \\
\hline BMI & $\mathrm{x}$ & $\mathrm{x}$ & $\mathrm{x}$ & $\mathrm{x}$ & $\mathrm{x}$ & $\mathrm{x}$ \\
\hline$w c$ & $x$ & $\mathrm{x}$ & $\mathrm{x}$ & $\mathrm{x}$ & $\mathrm{x}$ & $\mathrm{x}$ \\
\hline Blood pressure (SBP and DBP) & & $\mathrm{x}$ & $\mathrm{x}$ & $\mathrm{x}$ & $\mathrm{x}$ & $\mathrm{x}$ \\
\hline Insulin, glucagon, ghrelin & $x$ & & & & & $\mathrm{x}$ \\
\hline Glycated hemoglobin & $\mathrm{x}$ & & & $\mathrm{x}$ & $\mathrm{x}$ & $\mathrm{x}$ \\
\hline Lipids (TC, TG, HDL-c, LDL-c) & $\mathrm{x}$ & & $\mathrm{x}$ & $\mathrm{x}$ & $\mathrm{x}$ & $\mathrm{x}$ \\
\hline Serum glucose, urea and creatinine & $\mathrm{x}$ & & $\mathrm{x}$ & $\mathrm{x}$ & $\mathrm{x}$ & $\mathrm{x}$ \\
\hline $\begin{array}{c}\text { Serum sodium, calcium, potassium, } \\
\text { chloride }\end{array}$ & $x$ & & $x$ & $\mathrm{x}$ & $\mathrm{x}$ & $\mathrm{x}$ \\
\hline Urinalysis & $\mathrm{x}$ & & $\mathrm{x}$ & $\mathrm{x}$ & $x$ & $x$ \\
\hline $\begin{array}{l}\text { Pro-inflammatory cytokines (TNF-a, IL- } \\
\text { 6, Resistin) and anti-inflammatory } \\
\text { cytokines (IL-10, adiponectin) }\end{array}$ & $\mathrm{x}$ & & & & & $\mathrm{x}$ \\
\hline Appetite evaluation with VAS & & $\mathrm{x}$ & $\mathrm{x}$ & $\mathrm{x}$ & $\mathrm{x}$ & $\mathrm{x}$ \\
\hline$A E$ and $S E$ & & & $x$ & $x$ & $x$ & $x$ \\
\hline Adherence & & & $x$ & $\mathrm{x}$ & $\mathrm{x}$ & $\mathrm{x}$ \\
\hline
\end{tabular}

Fig. 1 Schedule of enrollment, interventions, and assessments in accordance with Standard Protocol Items: Recommendations for Interventional Trials (SPIRIT). M metformin, D/M dapagliflozin/metformin, BMI body mass index, WC waist circumference, TC total cholesterol, TG triglycerides, HDL-c high density cholesterol, LDL-c low density cholesterol, IL-6 interleukin-6, IL-10 interleukin-10, AE adverse event, SE secondary event 
Table 1 Inclusion and exclusion criteria

\begin{tabular}{|c|c|c|}
\hline Inclusion criteria & Exclusion criteria & Withdrawal criteria \\
\hline Age between 18 and 60 years & Current use of insulin or SU & Becoming pregnant during the study \\
\hline $\mathrm{BMI} \geq 40 \mathrm{~kg} / \mathrm{m}^{2}$ & Chronic kidney failure with eGFR $\leq 60 \mathrm{ml} / \mathrm{min} / 1.73 \mathrm{~m}^{2}$ & $\begin{array}{l}\text { Tobacco use (in the last month) (for the } \\
\text { secondary objectives) }\end{array}$ \\
\hline $\mathrm{HbA} 1 \mathrm{c}<9 \%$ & Use of loop diuretics & $\begin{array}{l}\text { Use of steroids drugs during the study } \\
\text { (for the secondary objectives) }\end{array}$ \\
\hline $\begin{array}{l}\text { Patients willing to participate and } \\
\text { that sign the informed consent letter }\end{array}$ & $\begin{array}{l}\text { Concomitant illnesses that predispose to volume } \\
\text { depletion, weight loss (including oncological diseases), } \\
\text { or metabolic acidosis (including drug users) }\end{array}$ & \\
\hline \multirow[t]{6}{*}{ Candidates to bariatric surgery } & History of recurrent genital or urinary tract infections & \\
\hline & $\begin{array}{l}\text { Current treatment with medications/products for } \\
\text { weight control }\end{array}$ & \\
\hline & $\begin{array}{l}\text { Untreated or uncontrolled hypothyroidism (defined } \\
\text { through TSH higher than } 4.2 \mathrm{\mu U} / \mathrm{ml} \text { ) }\end{array}$ & \\
\hline & Pregnant or lactating mother & \\
\hline & Tobacco use (in the last month) & \\
\hline & $\begin{array}{l}\text { Current use of steroids or nonsteroidal } \\
\text { anti-inflammatory drugs }\end{array}$ & \\
\hline
\end{tabular}

BMI body mass index, HbA1c glycated hemoglobin, SU sulfonylureas, eGFR estimated glomerular filtration rate, TSH thyroid stimulating hormone, CTCAE Common Terminology Criteria for Adverse Effects

of assessment. We do not expect dietary advice to influence our results because 1) the same nutritionist will assess all the participants, and 2) participants will be randomized. We will analyze data from those participants with lack of adherence to diet (defined as compliance of at least $80 \%$ of indicated diet) for the intention to treat analysis. The diet compliance will be assessed with concordance between the indicated foods (according to recommended kcals) and those reported by the participant using a 24-h recall with portion size estimation aids; these data will be collected for 3 days, including Sundays [25].

The nutritionist will determine the amount of aerobic exercise in minutes per week in baseline and follow-up visits. Participants are required to reach a minimum of 150 min per week of aerobic exercise during the baseline visit. The exercises are indicated specially for overweight people with limited mobility, and they can be performed during the day, with no special equipment required. The amount of exercise will be evaluated using a seven 24-h physical activity record, a week before each appointment [25]. Exercise will not be considered an inclusion criterion, but it will be evaluated post hoc as a confounding variable.

At the baseline and follow-up visits (months 1, 3, 6, and 12), the nutritionist will measure hunger through a visual analog scale (VAS). The participants will answer the question: How hungry do you feel? by making a mark on a $100 \mathrm{~mm}$ straight line, where the two extreme answers (not hungry or hungry) are anchored on opposite ends of the line [25]. Participants will be required to answer the VAS after being fed. For this purpose, we ask the participants to eat the breakfast recommended by the nutritionist.

A certified anthropometrist (certified by the International Society for the Advancement of Kinanthropometry) will evaluate weight using a scale with maximum weight capacity of $300 \mathrm{~kg}(662 \mathrm{lb})$ and precision of $100 \mathrm{~g}$ $(0.22 \mathrm{lb})$; height using a fixed stadiometer; WC using a fiberglass tape measure according to procedure manual for nutrition projects of the Center of Research in Nutrition and Health of the National Institute of Public Health of Mexico [26], and the procedure manual for assessment for clinic and anthropometric measures in adults and older adults of the Mexican Ministry of Health [27]. BMI will be calculated at screening, baseline, and follow-up visits. The percentage of excess body weight loss will be calculated using the following formula: \%EBWL = Initial BMI - Final BMI/Initial BMI Ideal BMI *100, and the percentage of weight loss will be calculated according to $\% \mathrm{WL}=$ Initial weight - Final weight/Initial weight * 100 in the follow-up visits. A trained nurse will evaluate the SBP and DBP by considering the mean average of two determinations taken 5 min apart, according to the procedure manual for assessment for clinic and anthropometric measures in adults and older adults of the Mexican Ministry of Health [27].

The following laboratory determinations will be performed at the screening visit and the follow-up visits at $1,3,6$, and 12 months: glucose, total cholesterol (TC), HDL-c, low-density lipoprotein cholesterol (LDL-c), TG, urea, creatinine, serum sodium, calcium, potassium and chloride, and urine analysis. HbA1c will be evaluated at the screening visit and at 3,6 , and 12 months. The 
measurement of insulin, glucagon, ghrelin, adiponectin, resistin, IL-6, and IL-10 will be performed at the baseline visit and at the appointment prior to surgery (depending on the response of each participant) or after a year (if weight loss is not achieved) using ELISA (enzyme linked immunosorbent assay).

\section{Evaluation of adherence and adverse effects}

One of the researchers not directly involved in the care of participating patients will provide the tablets needed for daily intake for a month and then the number of tablets required for visits in a sealed envelope. Adherence to medical treatment will be evaluated in follow-up visits after the run-in period. The participants will be asked to return their envelope and must consume at least $90 \%$ of the pills.

The adverse and secondary events will be recorded from the baseline visit and throughout the follow-up visits. Each adverse event will be evaluated by all the researchers and classified according to CTCAE version 5 . Accordingly, a grade 4 or 5 adverse event indicates discontinuation of treatment; grade 3 must be submitted to review by the medical team, and grades 1 or 2 require registration and intervention but not discontinuation of treatment. Adverse events grade 4 or 5 will be immediately reported to the ethics committee for follow-up and recommendations.

\section{Sample size}

Weight loss after dapagliflozin treatment has not been previously used as primary outcome in randomized controlled trials (RCT) of patients with prediabetes or diabetes and grade III obesity. Zhang et al. performed a meta-analysis that included seven RCTs evaluating the effect of metformin combined with SGLT2-inhibitors (dapagliflozin $10 \mathrm{mg} /$ day, canagliflozin $300 \mathrm{mg} /$ day, empagliflozin $25 \mathrm{mg} /$ day, or ipragliflozin $300 \mathrm{mg} /$ day) vs. metformin (at doses of 1.5 to $3 \mathrm{~g}$ /day) with placebo on HbA1c; fasting plasma glucose; and body weight over 24 weeks, 1 year, and 2 years. The total sample was 2847 patients with a mean BMI of $31.7 \pm 4.9 \mathrm{~kg} / \mathrm{m}^{2}$. After a year of treatment, the placebo-metformin control group showed a reduction in body weight of $1.1 \pm 3.39 \mathrm{~kg}$, whereas the SGLT-2 inhibitor-metformin group showed a $3.6 \pm 4.22 \mathrm{~kg}$ reduction with a mean difference of -2.6 $\mathrm{kg}(-3.17$ to $-2.03 \mathrm{~kg}), p<0.00001$ [16]. Using these data, we calculated a sample size using a mean difference formula. With a power of $80 \%$ (alpha level of 0.05 ) in a two-sided T-test, the sample size required is 90 patients: 45 patients in the $M$ group and 45 patients in the $\mathrm{D} / \mathrm{M}$ group. The final sample size required is 108 patients: 54 patients in metformin and 54 patients in the dapagliflozin group, allowing for a $20 \%$ dropout rate.

\section{Statistical analysis}

Quantitative variables will be presented as means and standard deviation or medians with interquartile ranges according to the data distribution. Categorical variables will be presented as frequencies or percentages. Intention-to-treat analyses will be applied as the primary analysis. The primary endpoint (weight loss at 12 months) will be analyzed using analysis of covariance (ANCOVA) while adjusting for baseline weight. We will also use a mixed-model regression analysis to estimate the treatment effect at $1,3,6$, and 12 months in other quantitative variables (due to the possibility of missing values), while considering as fixed effects the assigned treatment, sex, prediabetes or diabetes status, and random effects as the variability in the weight loss through the time. For qualitative variables analysis, we will use a mixed logistic regression analysis. A two-tailed $p$ value $<0.05$ is considered statistically significant. Statistical analysis will be performed using statistical packages: Statistical Package for the Social Sciences (SPSS) version 17.0 and STATA version 11.0.

\section{Trial management group, steering committee, monitoring, and data collection}

The trial management group consists of $\mathrm{MM}, \mathrm{AFH}$, CRR, and MAMA, and it is responsible of the design, data analysis, and publication of the trial. The primary investigators are $\mathrm{AFH}$ and MM.

Data will be collected and stored in properly codified individual files (VMZ, EEC, DMA, and APGM). Those documents are stored at the experimental sites in accordance with the rules and regulations of the Mexican Institute of Social Security (IMSS, Instituto Mexicano del Seguro Social). The study is monitored by the National Commission for Scientific Research (CNIC: Comisión Nacional de Investigación) of the IMSS. The National Commission of Scientific Research acts as a Trial Steering Committee. It is integrated by independent clinical and basic investigators (not affiliated to the same hospital or research center related with the investigators) that provide overall supervision and ensure that all registered trials throughout Mexico are conducted with rigorous standards. Those investigators are anonymous and randomly selected, and the evaluation process is through an electronic platform. A technical report informing the progress of the study must be presented to The National Commission for Scientific Research.

The Data Monitoring Committee consists of two investigators with expertise in methodology and statistics. They will receive interim reports and data summaries during the trial, monitor the data quality and participant safety, and assume responsibility for stopping the trial if necessary. The evaluations will be conducted every 6 months. 


\section{Publication and diffusion of results}

The results of the study will be submitted to international peer-reviewed journals, irrespective of its positive, negative, or inconclusive outcomes. Furthermore, the results will be presented at scientific conferences as abstracts, oral presentations, and posters.

The datasets generated and analyzed during the current study will be available from the corresponding author on reasonable request.

\section{Discussion}

Mexico has one of the highest prevalence rates of overweight and obesity worldwide. According to the National Health and Nutrition Survey, Mid Step (ENSANUT MC: Encuesta Nacional de Salud y Nutrición de Medio Camino 2016), 33.3\% of Mexicans older than 20 years are obese, with a prevalence of $27.7 \%$ in males and $38.6 \%$ in females. This survey also showed that the prevalence of class III obesity in males was $1.7 \%$ and $4.1 \%$ in females (2.4-fold higher in the latter) [28].

In 2008, a multidisciplinary clinic for the treatment of severe obesity and its related comorbidities was inaugurated at Hospital de Especialidades of Centro Médico Nacional Siglo XXI. One of the purposes of the Clinic is to offer bariatric surgery to qualifying patients, who are defined as those who achieve an appropriate weight loss (EBWL of 10\%). Several studies have proven that weight reduction prior to surgery decreases surgery-related complications and decreases hospitalization time [17]. Unfortunately, as previously observed in a quasiexperimental study performed at our Obesity Clinic, $46 \%$ of our patients do not achieve the required weight loss after a year of dietary treatment. Furthermore, $46 \%$ of our patients have prediabetes or T2D, $66 \%$ are hypertensive, and 33\% have dyslipidemia [29]. T2D treatments available in our clinic include metformin, pioglitazone, acarbose, and insulin (Neutral Protamine Hagedorn insulin, glargine and ultra-rapid insulin analogs). The patients with diabetes and obesity could benefit from the use of weight loss pharmacological treatment as recommended in 2015 by the Endocrine and Obesity Society [18]. However, Mexico's Federal Commission for the Protection against Sanitary Risks (COFEPRIS: Comisión Federal para la Protección contra Riesgos Sanitarios) has approved only two treatments: orlistat and liraglutide [30]. The former has a low adherence rate associated to its secondary effects, and the latter is not covered by social security due to its high cost. Therefore, we propose the use of a low-cost and secure treatment such as dapagliflozin for weight reduction in patients with grade III obesity and T2D or prediabetes. In our institution, the most common dose of metformin prescribed for glucose control is $850 \mathrm{mg}$ bid [31]. To assess if dapagliflozin has a higher efficacy, we decided to compare its addition to the usual treatment instead of using combination therapies such as Xigduo, which contains higher amounts of metformin (dapagliflozin $5 \mathrm{mg}$ with metformin 1000 $\mathrm{mg})$. Nowadays, both treatments are only indicated for glucose lowering in patients with diabetes or prediabetes. However, we aim to probe that beyond this effect, antihyperglycemic drugs such as metformin and dapagliflozin could also decrease weight in patients with higher grades of obesity.

Several studies have reported that metformin induces a modest decrease or no change, in weight [32, 33]. In an open randomized cross-over study, Hermann et al. reported a $2.64 \mathrm{~kg}$ loss of total weight (95\% CI -4.23 to $-1.05 \mathrm{~kg}$ ) after one year of treatment with metformin (patients with an average weight of $76.4 \mathrm{~kg}$ ) [34]. Similarly, in a quasi-experimental study, Stumvoll et al. observed a total weight loss of $2.7 \pm 1.3 \mathrm{~kg}$ in a study conducted in $10 \mathrm{~T} 2 \mathrm{D}$ patients with an average BMI of $32.1 \pm 3.2 \mathrm{~kg} / \mathrm{m}^{2}$ and treated with metformin at a dose of $2550 \mathrm{mg}$ ( $850 \mathrm{mg}$ three times a day) [35]. In addition, several studies have demonstrated that dapagliflozin effectively induces weight loss, decreases WC, reduces systolic and diastolic blood pressure as well as TG, and increases HDL-c both as monotherapy and in combination with other antidiabetic medications [10-15]. No studies have been conducted in patients with a BMI higher than $40 \mathrm{~kg} / \mathrm{m}^{2}$ to assess the weight loss capacity of the combination of $\mathrm{D} / \mathrm{M}$.

In their systematic review and meta-analyses, Zhang et al. calculated that a urinary glucose excretion of 71.2 $\mathrm{g} / 24 \mathrm{~h}$ induced by iSGLT2 should decrease $3.05 \mathrm{~kg}$. However, only a $2.5 \mathrm{~kg}$ weight reduction was achieved, representing 20\% less than expected [16]. This result led to Devenny et al. evaluating possible compensatory mechanisms that attenuate the effect of dapagliflozin. In a group of DIO rats, orally administered dapagliflozin in different doses induced weight and fat mass loss in a dose-dependent way. In this study, the effect on weight and fat mass was greater in those animals with restricted food consumption. Furthermore, in the group of animals fed ad libitum, they observed an increase in appetite as of day 7 and throughout the study. They evaluated appetite as the amount of food consumed each day in grams multiplied by the caloric value of the diet. They concluded that the increased appetite decreases the weight loss capacity induced by dapagliflozin [9]. This conclusion has not been evaluated in humans. In our protocol, we propose to evaluate ghrelin concentration, as a surrogate indicator of appetite [36].

Additionally, few studies have evaluated the effect of dapagliflozin on the concentration of inflammatory cytokines. In a single-arm interventional study, Okamoto et al. observed that dapagliflozin administered for 12 weeks decreases highly sensitive CRP (hs-CRP) and 
increases adiponectin [37]. Once again, this study included patients with an average BMI of $32.7 \pm 6.5 \mathrm{~kg} / \mathrm{m}^{2}$. According to the effect of decreased weight and WC previously reported in patients with grade I and II obesity treated with dapagliflozin, we expect a decrease in resistin and IL- 6 concentrations and an increase in IL-10 and adiponectin concentrations.

Finally, we aim to investigate if dapagliflozin treatment increases insulin and glucagon secretion in patients with obesity. Chronic hyperglycemia leads to a defect in insulin production due to glucotoxicity. The lowering of glucose by dapagliflozin has been suggested to reduce glucotoxicity and improve insulin secretion [38]. Furthermore, the normalization of blood glucose levels as well as the decrease in HbA1c levels and weight reduction induced by dapagliflozin seems to be related with an improvement in insulin sensitivity [38]. Some studies suggest that dapagliflozin could increase endogenous glucose production due to an increase in glucagon secretion by exerting a direct effect on alpha cell function [39]. Those effects remain controversial in people with obesity.

\section{Trial status}

The study is currently recruiting and enrolling participants according to version 2 of the protocol in June 2019. Recruitment began on July 7, 2018, and the approximate date for completion of recruitment will be July 7, 2020.

\section{Supplementary information}

Supplementary information accompanies this paper at https://doi.org/10. 1186/s13063-020-4121-x.

Additional file 1. Checklist for the report of study protocols according SPIRIT.

\footnotetext{
Abbreviations

\%EBWL: Percentage of excess body weight loss; \%WL: Percentage of weight Ioss; ADA: American Diabetes Association; AMPK: Adenosine monophosphate-activated protein kinase; ANCOVA: Analysis of covariance; BMI: Body mass index; CNIC: Comisión Nacional de Investigación Científica; COFEPRIS: Comisión Federal para la Protección contra Riesgos Sanitarios; CTCAE: Common Terminology Criteria for Adverse Effects; D/ M: Dapagliflozin/metformin; DBP: Diastolic blood pressure; DIO: Diet-induced obese; eGFR: estimated glomerular filtration rate; ELISA: Enzyme linked immunosorbent assay; ENSANUT: Encuesta Nacional de Salud y Nutrición; FIS: Fondo de Investigación en Salud; HbA1c: Glycated hemoglobin; HDLc: High-density lipoprotein cholesterol; hsCRP: Highly sensitive C-reactive protein; IL-10: Interleukin-10; IL-6: Interleukin-6; IMSS: Instituto Mexicano del Seguro Social; IR: Insulin resistance; iSGLT2: Sodium-glucose cotransporter-2 inhibitors; LDL-c: Low-density lipoprotein cholesterol; M: Metformin; OGGT: Oral glucose tolerance test; OR: Odd ratio; RCT: Randomized controlled trial; RCT: Randomized controlled trials; SBP: Systolic blood pressure; SPIRIT: Standard Protocol Items: Recommendations for Interventional Trials; SPSS: Statistical Package for the Social Sciences; SU: Sulfonylureas; T2D: Type 2 diabetes; TC: Total cholesterol; TG: Triglycerides; TSH: Thyroid stimulating hormone; UTI: Urinary tract infections; VAS: Visual analog scale; WC: Waist circumference
}

\section{Acknowledgements}

This manuscript is submitted in partial fulfillment of the requirements for a Ph.D. degree (AFH) at the Programa de Doctorado en Ciencias Médicas, Universidad Nacional Autónoma de México.

\section{Authors' contributions}

AFH, MAMA, CRR, and MM participate in study concept and design, planned the analyses and manuscript preparation. EEC, DMG, and APGM assisted in further development of the protocol and manuscript preparation. VMZ planned the analyses. All authors read and approved the final manuscript.

\section{Funding}

This work is funded by Fondo de Investigación en Salud (FIS) of IMSS, grant number: FIS/IMSS/PROT/G17-2/1752. The funding body will not intervene in the design of the study, analysis of the data, or writing of the manuscript.

\section{Availability of data and materials}

The datasets used and analyzed during the current study will be available from the corresponding author on reasonable request.

\section{Ethics approval and consent to participate}

The protocol has been approved by the Research and Ethics Committee of the IMSS (CNIC registry number: R-2016-785-094). The procedures are in compliance with local ethical regulations, the Mexican Law of General Health regarding Health Research, the institutional regulations, and the Declaration of Helsinki of 1975 and its amendments. It is also in compliance with the currently approved international codes and norms regarding Good Clinical Practice in Clinical Investigation.

Each patient will sign an informed consent prior to enrollment. The patients will be invited personally when they attend their regular visits to the outpatient clinic. The principal investigator or one of the co-investigators will also sign the consent. The patient will be informed about the freedom to withdraw consent at any time during the investigation.

The study is sponsored by the IMSS.

\section{Competing interests}

AFH is the Associate Editor of the BMC Endocrine Disorders Journal. The remaining authors declare that they have no competing interests.

\section{Author details}

'Unidad de Investigación Médica en Enfermedades Endocrinas, Hospital de Especialidades Centro Médico Nacional Siglo XXI, Instituto Mexicano del Seguro Social, Cuauhtémoc 330, Doctores, 06720 Mexico City, Mexico. ${ }^{2}$ Servicio de Endocrinología, Hospital de Especialidades Centro Médico Nacional Siglo XXI, Instituto Mexicano del Seguro Social, Cuauhtémoc 330, Doctores, 06720 Mexico City, Mexico.

Received: 5 August 2019 Accepted: 30 January 2020

Published online: 14 February 2020

\section{References}

1. Fioretto P, Giaccari A, Sesti G. Efficacy and safety of dapagliflozin, a sodium glucose cotransporter 2 (SGLT2) inhibitor, in diabetes mellitus. Cardiovasc Diabetol. 2015;14(1):142.

2. Baker WL, Smyth LR, Riche DM, Bourret EM, Chamberlin KW, White WB. Effects of sodium-glucose co-transporter 2 inhibitors on blood pressure: a systematic review and meta-analysis. J Am Soc Hypertens. 2014;8(4):262-75.e9.

3. Jayawardene D, Ward GM, O'Neal DN, Theverkalam G, Maclsaac Al, Maclsaac RJ. New treatments for type 2 diabetes: cardiovascular protection beyond glucose lowering? Heart Lung Circ. 2014;23(11):997-1008.

4. Faerch $\mathrm{K}$, Amadid $\mathrm{H}$, Nielsen LB, Ried-Larsen M, Karstoft $\mathrm{K}$, Persson $\mathrm{F}$, et al. Protocol for a randomised controlled trial of the effect of dapagliflozin, metformin and exercise on glycaemic variability, body composition and cardiovascular risk in prediabetes (the PRE-D Trial). BMJ Open. 2017;7(5): e013802.

5. Vasilakou D, Karagiannis T, Athanasiadou E, Mainou M, Liakos A, Bekiari E, et al. Sodium-glucose cotransporter 2 inhibitors for type 2 diabetes: a systematic review and meta-analysis. Ann Intern Med. 2013;159(4):262-74.

6. Monami M, Nreu B, Zannoni S, Lualdi C, Mannucci E. Effects of SGLT-2 inhibitors on diabetic ketoacidosis: a meta-analysis of randomised controlled trials. Diabetes Res Clin Pract. 2017;130:53-60. 
7. Pryor R, Cabreiro F. Repurposing metformin: an old drug with new tricks in its binding pockets. Biochem J. 2015;471(3):307-22

8. Mogul HR, Peterson SJ, Weinstein BI, Zhang S, Southren AL. Metformin and carbohydrate-modified diet: a novel obesity treatment protocol: preliminary findings from a case series of nondiabetic women with midlife weight gain and hyperinsulinemia. Heart Dis. 2001;3(5):285-92.

9. Devenny JJ, Godonis HE, Harvey SJ, Rooney S, Cullen MJ, Pelleymounter MA. Weight loss induced by chronic dapagliflozin treatment is attenuated by compensatory hyperphagia in diet-induced obese (DIO) rats. Obesity (Silver Spring). 2012;20(8):1645-52.

10. Nauck MA, Del Prato S, Duran-Garcia S, Rohwedder K, Langkilde AM, Sugg J, et al. Durability of glycaemic efficacy over 2 years with dapagliflozin versus glipizide as add-on therapies in patients whose type 2 diabetes mellitus is inadequately controlled with metformin. Diabetes Obes Metab. 2014;16(11): 1111-20.

11. List JF, Woo V, Morales E, Tang W, Fiedorek FT. Sodium-glucose cotransport inhibition with dapagliflozin in type 2 diabetes. Diabetes Care. 2009;32(4): 650-7.

12. Rosenstock J, Vico M, Wei L, Salsali A, List JF. Effects of dapagliflozin, an SGLT2 inhibitor, on $\mathrm{HbA}(1 \mathrm{c})$, body weight, and hypoglycemia risk in patients with type 2 diabetes inadequately controlled on pioglitazone monotherapy. Diabetes Care. 2012;35(7):1473-8.

13. Orme M, Fenici P, Lomon ID, Wygant G, Townsend R, Roudaut M. A systematic review and mixed-treatment comparison of dapagliflozin with existing anti-diabetes treatments for those with type 2 diabetes mellitus inadequately controlled by sulfonylurea monotherapy. Diabetol Metab Syndr. 2014;6:73.

14. Jabbour SA, Hardy E, Sugg J, Parikh S. Dapagliflozin is effective as add-on therapy to sitagliptin with or without metformin: a 24-week, multicenter, randomized, double-blind, placebo-controlled study. Diabetes Care. 2014; 37(3):740-50.

15. Bolinder J, Ljunggren $\mathrm{O}$, Kullberg J, Johansson L, Wilding J, Langkilde AM, et al. Effects of dapagliflozin on body weight, total fat mass, and regional adipose tissue distribution in patients with type 2 diabetes mellitus with inadequate glycemic control on metformin. J Clin Endocrinol Metab. 2012; 97(3):1020-31.

16. Zhang Q, Dou J, Lu J. Combinational therapy with metformin and sodiumglucose cotransporter inhibitors in management of type 2 diabetes: systematic review and meta-analyses. Diabetes Res Clin Pract. 2014;105(3): 313-21.

17. Santo MA, Riccioppo D, Pajecki D, Cleva R, Kawamoto F, Cecconello I. Preoperative weight loss in super-obese patients: study of the rate of weight loss and its effects on surgical morbidity. Clinics (Sao Paulo). 2014; 69(12):828-34

18. Apovian $\mathrm{CM}$, Aronne $\mathrm{L}$, Bessesen $\mathrm{DH}$, McDonnell ME, Murad MH, Pagotto $U$, et al. Pharmacological management of obesity: an endocrine Society clinical practice guideline. J Clin Endocrinol Metab. 2015;100(2):342-62.

19. Elliott JP, Gray EL, Yu J, Kalarchian MA. Medication Use Among Patients Prior to Bariatric Surgery. Bariatr Surg Pract Patient Care. 2015;10(3):105-9.

20. American DA. 9. Pharmacologic Approaches to Glycemic Treatment: Standards of Medical Care in Diabetes-2019. Diabetes Care. 2019;42(Suppl 1):S90-S102.

21. American DA. 2. Classification and Diagnosis of Diabetes: Standards of Medical Care in Diabetes-2019. Diabetes Care. 2019;42(Suppl 1):S13-28.

22. Chan AW, Tetzlaff JM, Altman DG, Laupacis A, Gotzsche PC, Krle AJK, et al. SPIRIT 2013 Statement: defining standard protocol items for clinical trials. Rev Panam Salud Publica. 2015;38(6):506-14.

23. Urbaniak GC, Plous S. Research Randomizer (version 4.0). 2013. https://www randomizer.org/index.htm. Accesed 1 Aug 2019.

24. US Department of Health and Human Services. Common Terminology Criteria for Adverse Events (CTCAE) version 5.0. 2017. https://ctep.cancer. gov/protocolDevelopment/electronic_applications/docs/CTCAE_v5_Quick_ Reference_8.5×11.pdf. Accessed 28 May 2018.

25. Beechy L, Galpern J, Petrone A, Das SK. Assessment tools in obesity psychological measures, diet, activity, and body composition. Physiol Behav. 2012;107(1):154-71.

26. Shamah-Levy T, Villalpando-Hernández S, Rivera-Dommarco J, editors. Manual de Procedimientos para Proyectos de Nutrición. Cuernavaca: Instituto Nacional de Salud Pública; 2006.

27. Velázquez-Monroy O, Lara-Esqueda A, Tapia-Olarte F, Romo-López L, Carrillo-Toscano J, Colín-Cario M, Montes-Reyes G. Manual de
Procedimientos. Toma de medidas clínicas y antropométricas en el adulto y adulto mayor. 2002. http://www.salud.gob.mx/unidades/cdi/documentos/ DOCSAL7518.pdf. Accesed 28 May 2018.

28. Romero-Martinez M, Shamah-Levy T, Cuevas-Nasu L, Gomez-Humaran IM Gaona-Pineda EB, Gomez-Acosta LM, et al. Methodological design of the National Health and Nutrition Survey 2016. Salud Publica Mex. 2017;59(3): 299-305.

29. Sanchez-Ruiz KL, Ferreira-Hermosillo A, Molina-Ayala MA, Ramirez-Renteria C, Mendoza-Zubieta V. Evaluation of cardiovascular risk factors in obesity before and after bariatric surgery. Rev Med Inst Mex Seguro Soc. 2017;55(5): 556-67.

30. Ferreira-Hermosillo A, Salame-Khouri L, Cuenca-Abruch D. Pharmacological treatment of obesity. Rev Med Inst Mex Seguro Soc. 2018;56(4):395-409.

31. Perez-Cuevas R, Doubova SV, Suarez-Ortega M, Law M, Pande AH, Escobedo J, et al. Evaluating quality of care for patients with type 2 diabetes using electronic health record information in Mexico. BMC Med Inform Decis Mak. 2012;12:50.

32. DeFronzo RA, Goodman AM. Efficacy of metformin in patients with noninsulin-dependent diabetes mellitus. The Multicenter Metformin Study Group. N Engl J Med. 1995;333(9):541-9.

33. Goodarzi MO, Bryer-Ash M. Metformin revisited: re-evaluation of its properties and role in the pharmacopoeia of modern antidiabetic agents. Diabetes Obes Metab. 2005;7(6):654-65.

34. Hermann LS, Karlsson JE, Sjostrand A. Prospective comparative study in NIDDM patients of metformin and glibenclamide with special reference to lipid profiles. Eur J Clin Pharmacol. 1991;41(3):263-5.

35. Stumvoll M, Nurjhan N, Perriello G, Dailey G, Gerich JE. Metabolic effects of metformin in non-insulin-dependent diabetes mellitus. N Engl J Med. 1995; 333(9):550-4

36. Nowak A, Czkwianianc E. A contemporary approach to body mass regulation mechanisms. Prz Gastroenterol. 2016;11(2):73-7.

37. Okamoto A, Yokokawa H, Sanada H, Naito T. Changes in levels of biomarkers associated with adipocyte function and insulin and glucagon kinetics during treatment with dapagliflozin among obese type 2 diabetes mellitus patients. Drugs R D. 2016;16(3):255-61.

38. Scheen AJ, Paquot N. Metabolic effects of SGLT-2 inhibitors beyond increased glucosuria: A review of the clinical evidence. Diabetes Metab. 2014;40(6 Suppl 1):S4-S11.

39. Bonner C, Kerr-Conte J, Gmyr V, Queniat G, Moerman E, Thevenet J, et al. Inhibition of the glucose transporter SGLT2 with dapagliflozin in pancreatic alpha cells triggers glucagon secretion. Nat Med. 2015;21(5):512-7.

\section{Publisher's Note}

Springer Nature remains neutral with regard to jurisdictional claims in published maps and institutional affiliations.

Ready to submit your research? Choose BMC and benefit from:

- fast, convenient online submission

- thorough peer review by experienced researchers in your field

- rapid publication on acceptance

- support for research data, including large and complex data types

- gold Open Access which fosters wider collaboration and increased citations

- maximum visibility for your research: over $100 \mathrm{M}$ website views per year

At BMC, research is always in progress.

Learn more biomedcentral.com/submissions 